\title{
Alendronate Improves Low Bone Mineral Density Induced by Steroid Therapy in Crohn's Disease
}

\author{
Tomoyuki Tsujikawa ${ }^{1-3}$, Akira Andoh ${ }^{1-3}$, Osamu Inatomi ${ }^{1-3}$, Shigeki Bamba ${ }^{1-3}$, \\ Tamio Nakahara ${ }^{1-3}$, Masaya Sasaki ${ }^{1-3}$, Haruyasu Saito ${ }^{1-3}$ and Yoshihide Fujiyama ${ }^{1-3}$
}

\begin{abstract}
Aim We investigated whether steroid therapy for Crohn's disease (CD) patients influences bone mineral density (BMD), and whether alendronate is effective for improving this loss of BMD.

Methods We recruited 16 outpatients with CD. The BMD of the whole body, the lumbar spine, and the proximal femoral neck was measured by dual-energy X-ray absorptimetry. The BMD was expressed as a $\mathrm{T}$ score. Some CD patients with low BMD values had been given vitamin K2 or alendronate for one year.

Results In the steroid-dependent group, the mean dose of prednisolone was $968 \mathrm{mg}$ per year and $2.7 \mathrm{mg}$ per day. Although the duration of the disease was not related to the $\mathrm{T}$ score, the amount of total steroids was negatively correlated with the $\mathrm{T}$ score among patients taking no preventative drugs. The $\mathrm{T}$ score in the vitamin $\mathrm{K} 2$ group after one year did was not altered in the 3 areas examined. On the other hand, the $\mathrm{T}$ score in the alendronate group increased by $2.8 \%$ for the whole body, $4.5 \%$ in the lumbar spine, and $3.4 \%$ in the proximal femoral neck.

Conclusion The BMD of Japanese CD patients was decreased depending on the total amount of steroid administered, and oral alendronate improved the loss of BMD.
\end{abstract}

Key words: Crohn's disease, bone mineral density, steroid, alendronate

(Inter Med 48: 933-937, 2009)

(DOI: 10.2169/internalmedicine.48.2005)

\section{Introduction}

Loss of bone mineral density (BMD) has been reported as a common complication of inflammatory bowel disease (IBD) in Western countries, and patients with Crohn's disease (CD) have an especially high risk of developing osteoporosis (1). In Japan, there have been several studies on the complications induced by steroid therapy in ulcerative colitis (UC) (2-4). Patients with UC who received more than $10,000 \mathrm{mg}$ of steroids are recommended to undergo a total colectomy before any osteoporotic manifestations develop. However, there are few reports on the relationship between the loss of BMD (5) and the effects of steroid therapy in Japanese patients with CD. Since the treatment for CD in Japan has shifted from nutritional therapy to drug therapy, including steroids, there is an increasing focus on the bone complications from steroids. Although the general guidelines for steroid-induced osteoporosis have been published (6), the prevention and optimal treatment for bone disease in patients with CD still needs to be established. As a secondgeneration bisphosphonate drug, alendronate is a potent and selective inhibitor of osteoclasts (7). It has been reported that alendronate therapy significantly increases the BMD in CD patients in Europe and the USA $(8,9)$, but there have been no reports in Japan. Therefore, we investigated whether steroid therapy for Japanese CD patients influences the $\mathrm{BMD}$, and whether alendronate is effective in improving this loss of BMD.

\footnotetext{
${ }^{1}$ Department of Internal Medicine, Shiga University of Medical Science, Otsu, ${ }^{2}$ Division of Clinical Nutrition, Shiga University of Medical Science Hospital, Otsu and ${ }^{3}$ Department of Endoscopic Medicine, Shiga University of Medical Science Hospital, Otsu

Received for publication January 3, 2009; Accepted for publication February 24, 2009

Correspondence to Dr. Tomoyuki Tsujikawa, tujikawa@belle.shiga-med.ac.jp
} 


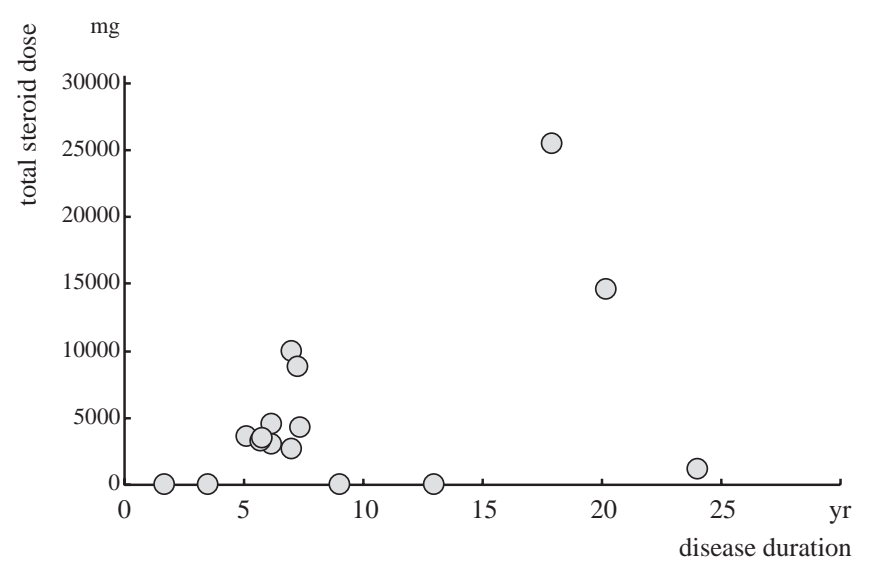

Figure 1. Relationship between the total steroid dose and disease duration in outpatients with Crohn's disease ( $n=16)$.

\section{Methods}

\section{Patients}

This study was carried out at the Hospital of Shiga University of Medical Science in April 2005. We recruited 16 outpatients with $\mathrm{CD}$ (11 males and 5 females; mean age 33.4 years, range 20-54 years; mean duration of disease 9.7 years, range 1.7-20.2 years). None of the female patients were menopausal. Fifteen of these patients had been utilizing home enteral nutrition (HEN) (600-1,500 kcal/day) for more than 6 months. Twelve had been taking prednisolone. All 16 patients were taking salicylates, and three were taking AZT/6MP. None of the patients were treated by the periodic administration of infliximab in this study.

\section{Measurement of bone mineral density (BMD)}

The BMD of the whole body, the lumbar spine (L2-L4), and the proximal femoral neck was measured by dualenergy X-ray absorptimetry (DEXA) using a Lunar instrument (GE Medical Systems Lunar Corp., Madison, WI, USA). The BMD was also expressed as a SD score (z score), and as a T score. The $\mathrm{T}$ score was calculated according to the following formula:

$\mathrm{T}$ Score $=$

$\underline{\text { ( }\{\text { Measured BMD }\}-\{\text { Mean Peak BMD of Gender-Matched Control }\})}$

(Standard Deviation of Peak BMD of Gender-Matched Control)

The $\mathrm{T}$ score expresses the deviation in the BMD relative to the peak BMD measured by the standard deviation. The reference population consisted of a healthy Japanese population. Osteopenia was defined according to the recommendations of the World Health Organization as a $\mathrm{T}$ score of less than -1.0 , and osteoporosis as a $\mathrm{T}$ score of $<-2.5$.

\section{Treatment}

Nine patients whose $\mathrm{T}$ scores were less than 0 , which included one patient who had never used steroids, were given $45 \mathrm{mg}$ of vitamin K2 (menatetrenone) daily for more than
Table 1. Comparison of Patients with Low and Normal BMD Values

\begin{tabular}{lcc} 
& $\begin{array}{c}\text { Low BMD } \\
(\text { T score }<0)\end{array}$ & $\begin{array}{c}\text { Normal BMD } \\
(\text { T score }>0)\end{array}$ \\
\hline number & 10 & 6 \\
Gender(m/f) & $6 / 4$ & $5 / 1$ \\
Age $(\mathrm{yr})$ & $32.5 \pm 9.7$ & $35.0 \pm 8.2$ \\
Duration(yr) & $10.1 \pm 5.5$ & $9.2 \pm 8.2$ \\
BMI $\left(\mathrm{kg} / \mathrm{m}^{2}\right)$ & $19.3 \pm 2.5$ & $21.7 \pm 2.9$ \\
Resection(n) & $6 / 10$ & $3 / 6$ \\
Smoking(n) & $2 / 10$ & $1 / 6$ \\
CDAI(points) & $128 \pm 74$ & $110 \pm 70$ \\
Steroid dose(mg) & $9320 \pm 8331$ & $1223 \pm 1540^{*}$ \\
& &
\end{tabular}

BMD; bone mineral density, *; $\mathrm{p}<0.05$

one year. All patients were also recommended to take a daily supplement of calcium L-aspartate unless their dietary calcium intake exceeded 1,000 mg/day. Since alendronate was made available, 5 patients who had been taking steroids ceased taking vitamin $\mathrm{K} 2$, and one new steroid user whose $\mathrm{T}$ score was less than 0 started to receive $5 \mathrm{mg}$ of alendronate daily.

\section{Biochemical markers of bone metabolism}

In five out of the 6 steroid user-patients undergoing alendronate treatment, serum and urine samples were obtained for the measurement of biochemical markers of bone turnover. Serum bone-specific alkaline phosphatase (BAP) as a marker of bone formation was measured by an enzyme immunoassay. The urinary excretion of cross-liked Ntelopeptides from type X collagen (NTx) corrected for the creatinine concentration was used as a maker of bone resorption, and was measured by an enzyme-linked immunosorbent assay.

\section{Statistical analysis}

The results are expressed as means \pm SD. Comparisons between group means were performed using student's unpaired t-test or a chi-squared test, as appropriate, and the slopes were evaluated using regression methods. A p value < 0.05 was taken to be statistically significant.

\section{Results}

The amount of total steroids (as prednisolone) and the duration of disease among the patients with $\mathrm{CD}$ is shown in Fig. 1. There are two main groups of patients receiving steroids treatment. One is the steroid-never user (4 patients), and the other is the steroid-dependent user (12 patients). Among the steroid-dependent group, the mean dose of prednisolone was $968 \mathrm{mg} /$ year and $2.7 \mathrm{mg} /$ day.

According to the $\mathrm{T}$ score of the whole body, the patients were divided into 2 groups with $\mathrm{T}$ scores less than 0 (low BMD group), and above 0 (normal BMD group) (Table 1). Between these 2 groups, the parameters of gender, age, disease duration, Crohn's disease activity index (CDAI), body 
a

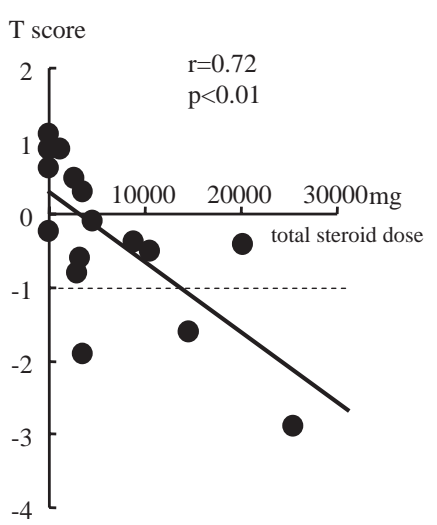

b

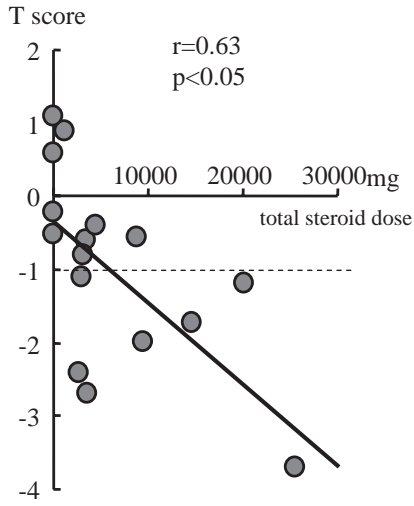

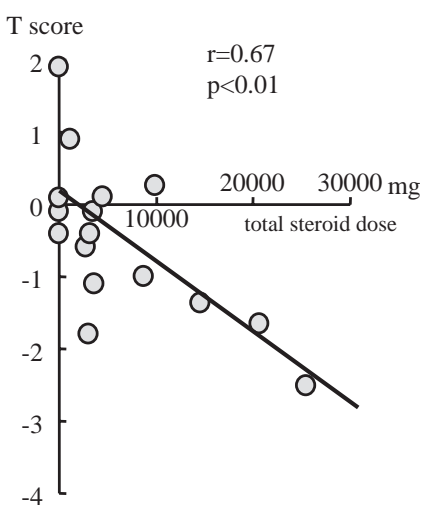

Figure 2. Relationship between the total steroid dose and T score. a: whole body, b: lumbar spine (L2-L4), and c: proximal femoral neck. The linear line is fitted by regression methods ( $=16)$.

Table 2. Background of Vitamin K2 or Alendronate Groups

\begin{tabular}{lcc} 
& vitamin K2 & alendronate \\
\hline number & 9 & 6 \\
Gender(m/f) & $5 / 4$ & $4 / 2$ \\
Age(yr) & $33.1 \pm 9.4$ & $29.2 \pm 5.7$ \\
Duration(yr) & $10.3 \pm 6.0$ & $10.3 \pm 6.3$ \\
BMI $\left(\mathrm{kg} / \mathrm{m}^{2}\right)$ & $19.3 \pm 2.6$ & $19.1 \pm 2.5$ \\
Resection(n) & $5 / 9$ & $4 / 6$ \\
Smoking(n) & $2 / 9$ & $1 / 6$ \\
CDAI(points $)$ & $125 \pm 74$ & $123 \pm 74$ \\
Steroid user & 8 & 6 \\
Steroid dose $(\mathrm{mg})$ & $9378 \pm 8334$ & $10250 \pm 6220$ \\
BMD whole body $\left(\mathrm{g} / \mathrm{cm}^{2}\right)$ & $1.08 \pm 0.13$ & $1.08 \pm 0.12$ \\
BMD lumber spine $\left(\mathrm{g} / \mathrm{cm}^{2}\right)$ & $0.98 \pm 0.15$ & $0.97 \pm 0.12$ \\
BMD femoral neck $\left(\mathrm{g} / \mathrm{cm}^{2}\right)$ & $0.82 \pm 0.14$ & $0.79 \pm 0.11$ \\
& & \\
\hline
\end{tabular}

There are no statistically differences between vitamin K2 and alendronate groups

mass index (BMI), small bowel resection, and smoking showed no statistically significant differences, but the total amount of steroids in the low BMD group was significantly higher than that in the normal BMD group. Among the 4 steroid-never users, only one patient belonged to the low BMD group. We calculated the relationship between the total amount of steroids and the BMD value before vitamin K2 or alendronate administration. Although the duration of disease was not related to the $\mathrm{T}$ score (data not shown), the amount of total steroids was negatively correlated with the $\mathrm{T}$ score (Fig. 2). The estimated total dose of steroids at $\mathrm{T}$ scores of below -1.0 and -2.5 were $12,800 \mathrm{mg}$ and 27,300 $\mathrm{mg}$, respectively, in the whole body, 6,700 $\mathrm{mg}$ and 20,600 $\mathrm{mg}$ in the lumbar spine (L2-L4), and 11,600 $\mathrm{mg}$ and 27,500 $\mathrm{mg}$ in the proximal femoral neck.

The backgrounds of patients in the vitamin K2 versus alendronate groups (Table 2) did not differ significantly including BMD values before treatment. The \% changes in the $\mathrm{T}$ score after one year among the $\mathrm{CD}$ patients taking vitamin K2 or alendronate are shown in Fig. 3. The mean $\mathrm{T}$ score in the vitamin $\mathrm{K} 2$ group did not change in the 3 regions examined after one year. However, the value of the

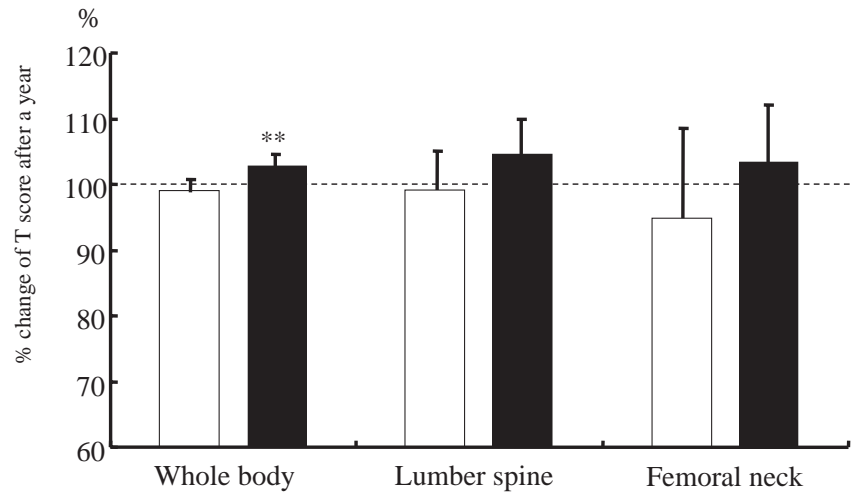

Figure 3. Percentage change in the $\mathbf{T}$ score from baseline over $1 \mathrm{yr}$ for the whole body, lumbar spine L2-L4, and left femoral neck in patients taking vitamin K2 (white bar, $n=9$ ) or alendronate (black bar, $n=6$ ). The values represent means \pm SD. ${ }^{* *} \mathbf{p}<0.01$ between baseline and after one year.

whole body in the alendronate group significantly increased after one year. Both vitamin $\mathrm{K} 2$ and alendronate did not have a harmful influence on intestinal inflammation in CD patients. Among those patients taking steroid and alendro- 

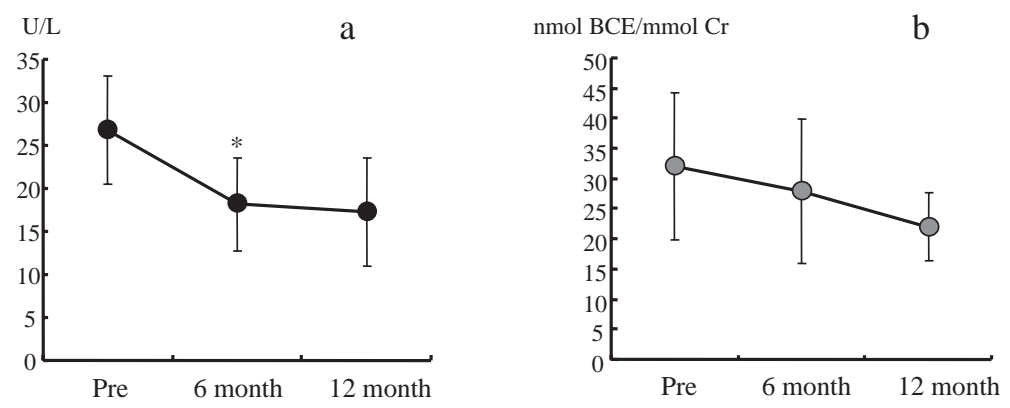

Figure 4. Changes in the serum BAP (a) and NTx (b) levels during alendronate treatment. The data values are expressed as means \pm SD. $n=5$. *p<0.05 between pre-treatment and 6 months of treatment.

nate, the serum BAP and urine NTx levels before and at 6 months and 12 months after administration were evaluated in 5 patients. Both the BAP and NTx levels decreased gradually, and the BAP value after 6 months of administration was significantly reduced as compared to before the administration of alendronate (Fig. 4).

\section{Discussion}

The present study showed for the first time that steroid therapy decreased the BMD in a dose-dependent manner in Japanese CD patients. The number of patients in this study was only 16 . There were more than 50 outpatients in our hospital in April 2005. However, some patients referred to our hospital had no records of their total steroid dose and BMD. In some other patients, steroids were administered previously, but they were steroid-free at that time. Finally, only 16 patients had been taking steroids and had their BMD checked periodically. Previous studies have reported that CD itself is one of the risk factors for osteoporosis (10). We could not arrive at the same conclusion, because only 4 patients without steroid administration had their BMD measured. The pathogenesis of osteoporosis in CD patients is considered to be mutifactorial. Many studies have indicated that cumulative steroid use, disease duration, low BMI, calcium and vitamin D deficiency, small bowel involvement or resection, smoking, gender and increasing age are associated with a loss of BMD (11-17). In this study, most patients had been on nutritional therapy, which means that most patients were supported by a balanced caloric diet, and nutritional deficiencies would be unlikely to occur. Recently, an analysis from the TREAT study in the USA showed that steroid therapy for CD patients exerts a harmful effect on the prognosis, including being prone to infection (18). Therefore, it has been suggested that the use of steroids should be cut down from the viewpoint of other adverse effects. Previous studies suggested more than $10,000 \mathrm{mg}$ of total steroid use increased the risk of adverse effects in IBD patients (19). Our study indicated more than $20,000 \mathrm{mg}$ of steroids resulted in a great risk of osteoporosis ( $\mathrm{T}$ score $<-2.5$ ) in $\mathrm{CD}$ patients. It is important to note that around $10,000 \mathrm{mg}$ of total steroids generally averts any irreversible reactions for $\mathrm{CD}$ patients using steroids, although the same finding for UC patients has been emphasized in Japan.

With respect to therapy for the loss of BMD, alendronate administration for one year in $\mathrm{CD}$ patients improved their BMD in accordance with improvements in their BAP and NTx values. These results suggested that the therapeutic guideline for steroid users (20) should also apply to CD patients. However, this drug should be carefully administered, because many female patients with $\mathrm{CD}$ are of reproductive age. Our data did not support the use of vitamin K2 for improving the loss of $\mathrm{BMD}$ in $\mathrm{CD}$ patients. It has been reported that vitamin $\mathrm{K} 2$ stimulates osteoblastosis and inhibits osteoclastogenesis in vitro (21), and also attenuates the loss of BMD induced by steroids in a rat model (22). On the other hand, the effects of vitamin $\mathrm{K} 2$ on the steroid-induced loss of BMD in humans has been unclear. Sasaki et al showed that simultaneous vitamin $\mathrm{K} 2$ administration partially inhibited the steroid-induced loss of BMD in patients with chronic glomerulonephritis, and that it acted via preventing the reduction of osteoprotegerin (23). However, the mean BMD in that study gradually decreased during one year, even in the group co-administered with vitamin K2. Although we did not set a negative control group that did not receive any effective drugs for osteoporosis, vitamin $\mathrm{K} 2$ may partly improve the loss of BMD induced by steroids, because the mean BMD in the vitamin $\mathrm{K} 2$ group did not decrease during one year. No patients taking vitamin K2 or alendronate experienced any bone fractures, which is the final endpoint for the loss of BMD, during the observation period. In this study, patients receiving periodic infliximab were excluded because the steroids were tapered and finally terminated once these patients started maintenance therapy with infliximab. TNF- $\alpha$ not only modulates the intestinal inflammation of CD patients, but has also been reported to inhibit osteoblast differentiation and to increase osteoclastogenesis through the activation of the p55 tumor necrosis factor receptor (24). Recent reports have demonstrated that maintenance infliximab treatment is associated with an improved BMD in $\mathrm{CD}$ patients (25). Therefore, replacing steroid-dependent therapy to infliximab therapy with steroid termination is strongly desirable from the viewpoint of preventing BMD loss. 
In conclusion, the BMD of Japanese CD and UC patients decreased depending on the total amount of steroids admin- istered, and the oral administration of steroid and alendronate improved this loss of BMD induced by the steroids.

\section{References}

1. Adachi JD, Rostom A. Metabolic bone disease in adults with inflammatory bowel disease. Inflamm Bowel Dis 5: 200-211, 1999.

2. Kusunoki M, Möeslein G, Shoji Y, et al. Steroid complications in patients with ulcerative colitis. Dis Colon Rectum 35: 1003-1009, 1992.

3. Uchida K, Araki T, Toiyama Y, et al. Preoperative steroid-related complications in Japanese pediatric patients with ulcerative colitis. Dis Colon Rectum 49: 74-79, 2006.

4. Shinozaki M, Suzuki K, Sawada T, Tsuno N, Higuchi Y, Muto T. Steroid complications and surgery in intractable ulcerative colitis. J Gastroenterol 33: 196-200, 1998.

5. Kunisaki R, Chaki O, Taguchi T, Sugiyama T, Sugita A. Infliximab and the bone in Crohn's disease. Aliment Pharmacol Ther 21: 789-790, 2005.

6. Soen S, Tanaka Y. Glucocorticoid-induced osteoporosis: skeletal manifestations of glucocorticoid use and 2004 Japanese Society for Bone and Mineral Research-proposed guidelines for its management. Mod Rheumatol 15: 163-168, 2005.

7. Plotkin LI, Weinstein RS, Parfitt AM, Roberson PK, Manolagas SC, Bellido T. Prevention of osteocyte and osteoblast apoptosis by bisphosphonates and calcitonin. J Clin Invest 104: 1363-1374, 1999.

8. Bailen LS. Bones and Crohn's: should we treat Crohn's disease patients with alendronate? Inflamm Bowel Dis 7: 175-176, 2001.

9. Haderslev KV, Tjellesen L, Sorensen HA, Staun M. Alendronate increases lumbar spine bone mineral density in patients with Crohn's disease. Gastroenterology 119: 639-646, 2000.

10. Ghosh S, Cowen S, Hannan WJ, Ferguson A. Low bone mineral density in Crohn's disease, but not in ulcerative colitis, at diagnosis. Gastroenterology 107: 1031-1039, 1994.

11. Pollak RD, Karmeli F, Eliakim R, Ackerman Z, Tabb K, Rachmilewitz D. Femoral neck osteopenia in patients with inflammatory bowel disease. Am J Gastroenterol 93: 1483-1490, 1998.

12. Schulte C, Dignass AU, Mann K, Goebell H. Reduced bone mineral density and unbalanced bone metabolism in patients with inflammatory bowel disease. Inflamm Bowel Dis 4: 268-275, 1998.

13. Compston JE, Judd D, Crawley EO, et al. Osteoporosis in patients with inflammatory bowel disease. Gut 28: 410-415, 1987.

14. Robinson RJ, al-Azzawi F, Iqbal SJ, et al. Osteoporosis and deter- minants of bone density in patients with Crohn's disease. Dig Dis Sci 43: 2500-2506, 1998.

15. Bischoff SC, Herrmann A, Göke M, Manns MP, von zur Mühlen A, Brabant G. Altered bone metabolism in inflammatory bowel disease. Am J Gastroenterol 92: 1157-1163, 1997.

16. Andreassen H, Hylander E, Rix M. Gender, age, and body weight are the major predictive factors for bone mineral density in Crohn's disease: a case-control cross-sectional study of 113 patients. Am J Gastroenterol 94: 824-848, 1999.

17. Silvennoinen JA, Lehtola JK, Niemelä SE. Smoking is a risk factor for osteoporosis in women with inflammatory bowel disease. Scand J Gastroenterol 31: 367-371, 1996.

18. Lichtenstein GR, Feagan BG, Cohen RD, et al. Serious infections and mortality in association with therapies for Crohn's disease: TREAT registry. Clin Gastroenterol Hepatol 4: 621-630, 2006.

19. Silvennoinen JA, Karttunen TJ, Niemelä SE, Manelius JJ, Lehtola JK. A controlled study of bone mineral density in patients with inflammatory bowel disease. Gut 37: 71-76, 1995.

20. Soen S. Guidelines for the treatment of glucocorticoid-induced osteoporosis and their future problems. Clin Calcium 16: 1788-1796, 2006.

21. Koshihara Y, Hoshi K. Vitamin K2 enhances osteocalcin accumulation in the extracellular matrix of human osteoblasts in vitro. $\mathbf{J}$ Bone Miner Res 12: 431-438, 1997.

22. Hara K, Kobayashi M, Akiyama Y. Vitamin K2 (menatetrenone) inhibits bone loss induced by prednisolone partly through enhancement of bone formation in rats. Bone 31: 575-581, 2002.

23. Sasaki N, Kusano E, Takahashi $\mathrm{H}$, et al. Vitamin $\mathrm{K} 2$ inhibits glucocorticoid-induced bone loss partly by preventing the reduction of osteoprotegerin (OPG). J Bone Miner Metab 23: 41-47, 2005.

24. Abu-Amer Y, Erdmann J, Alexopoulou L, Kollias G, Ross FP, Teitelbaum SL. Tumor necrosis factor receptors types 1 and 2 differentially regulate osteoclastogenesis. J Biol Chem 275: 2730727310, 2000.

25. Bernstein M, Irwin S, Greenberg GR. Maintenance infliximab treatment is associated with improved bone mineral density in Crohn's disease. Am J Gastroenterol 100: 2031-2035, 2005.

(C) 2009 The Japanese Society of Internal Medicine http://www.naika.or.jp/imindex.html 